PROCEEDINGS OF THE

AMERICAN MATHEMATICAL SOCIETY

Volume 130, Number 8 , Pages 2203-2213

S 0002-9939(02)06561-9

Article electronically published on February 27, 2002

\title{
THE UNIVERSAL NORM DISTRIBUTION AND SINNOTT'S INDEX FORMULA
}

\author{
YI OUYANG
}

(Communicated by David E. Rohrlich)

\begin{abstract}
We define and study the universal norm distribution in this paper, which generalizes the well studied universal ordinary distribution by Kubert (1979). We display a resolution of Anderson type for the universal norm distribution. Furthermore, we prove a general index formula between different universal norm distributions. As a special case, this general index formula recovers the hard calculation in Sinnott's Annals paper (1978).
\end{abstract}

\section{INTRODUCTION}

In his famous paper [7, Sinnott successfully obtained the index formulas of Stickelberger ideal and circular units in cyclotomic fields, which generalized the results of Kummer and Iwasawa. Let $G_{r}=\operatorname{Gal}\left(\mathbb{Q}\left(\mu_{r}\right) / \mathbb{Q}\right)$ and $R=\mathbb{Z}\left[G_{r}\right]$. Let $c$ be the complex conjugation and let $J=\{1, c\}$. For any ideal $\theta \in R$ and a given $R$-module $M$, let $M^{\theta}$ be the submodule of $M$ annihilated by $\theta$. In [7], Sinnott introduced a $G_{r}$-lattice $U$ inside $\mathbb{Q}\left[G_{r}\right]$, which we call Sinnott's module. Sinnott's index calculation in [7, in a large part, is the calculation of the indices $\left(R^{\theta}: U^{\theta}\right)$ for $\theta=(0)$ and $\theta=(1+c)$ and of the cohomology group $\hat{H}^{*}(J, U)$.

Sinnott's module $U$ has been observed by Kubert [4] as a realization of the universal ordinary distribution $U_{r}$. Recently Anderson (see Appendix of Ouyang [6] discovered a resolution $\left(L_{r}^{\bullet}, d\right)$ for $U_{r}$ where $L_{r}^{\bullet}$ is a torsion free finite graded $G_{r^{-}}$ module and $d$ is $G_{r}$-compatible. We call $L_{r}^{\bullet}$ Anderson's module for $U_{r}$.

We develop the general theory of universal norm distributions in this paper, with $U_{r}$ and $R$ as special cases. Each universal norm distribution $U_{\mathbf{n}, r}$ is shown to have properties similar to those of $U_{r}$. In particular, Anderson's module $L_{r}^{\bullet}$, equipped with a $G_{r}$-compatible differential $d_{\mathbf{n}}$, is a resolution for $U_{\mathbf{n}, r}$ (Theorem 3.3). As an application, we compute the $J$-cohomology of $U_{\mathbf{n}, r}$ in Proposition 3.6 Our approach gives simpler proofs of Kubert's classical results in [4, 5].

For the space $V_{r}^{\bullet}=\mathbb{Q} \otimes L_{r}^{\bullet}$, we find a connecting isomorphism $\phi_{\mathbf{n}_{1}, \mathbf{n}_{2}, r}$ between $\left(V_{r}^{\bullet}, d_{\mathbf{n}_{1}}\right)$ and $\left(V_{r}^{\bullet}, d_{\mathbf{n}_{2}}\right)$ for any two differentials $d_{\mathbf{n}_{1}}$ and $d_{\mathbf{n}_{2}}$. Through this isomorphism, $\phi_{\mathbf{n}_{1}, \mathbf{n}_{2}, r} U_{\mathbf{n}_{1}, r}$ is a lattice in $\mathbb{Q} \otimes U_{\mathbf{n}_{2}, r}$. In the special case of $U_{r}$ and $R$, Sinnott's module $U$ is exactly the image of $U_{r}$ in $\mathbb{Q}\left[G_{r}\right]$. In Theorem 4.1, we prove a general index formula (4.1) for the index $\left(U_{\mathbf{n}_{2}, r}^{\theta}: \phi_{\mathbf{n}_{1}, \mathbf{n}_{2}, r} U_{\mathbf{n}_{1}, r}^{\theta}\right)$ for $\theta$ an arbitrary ideal in $R$. Moreover, the terms in the general formula (4.1) are interpreted as the

Received by the editors February 25, 2001.

2000 Mathematics Subject Classification. Primary 11R18; Secondary 11R27, 11R34, 18G40. 
orders of $E_{2}$-terms of certain spectral sequences. With our previous calculation of the $J$-cohomology of $U_{\mathbf{n}, r}$, we are able to recover the indices of Sinnott.

The universal norm distribution is actually quite common in number theory. For example, the integer ring of $\mathbb{Q}\left(\mu_{r}\right)$ is also one of the universal norm distributions. Moreover, in a separate paper, we are going to study the universal Euler system, which is also a special type of universal norm distributions. Furthermore, without any extra difficulty, one can develop the theory of universal norm distributions and handle the index calculation in the function field case.

This paper is based on the working note 2 of my advisor, Professor Greg W. Anderson. The material which is discussed in $\S 2$ of this paper is essentially from 2]. I also benefited greatly from numerous discussions with him. I thank him sincerely. This paper was finished when I was visiting I.H.E.S. I thank them for their hospitality. I also thank the referee for many helpful comments.

\section{The Regulator map $\operatorname{reg}(A, B, \lambda)$}

2.1. Sinnott's symbol. Let $A$ and $B$ be lattices in a finite dimensional vector space $V$ over $\mathbb{F}$ where $\mathbb{F}=\mathbb{Q}$ or $\mathbb{R}$. Necessarily there exists some $\mathbb{F}$-linear automorphism $\phi$ of $V$ such that $\phi(A)=B$. Put

$$
(A: B)_{V}:=|\operatorname{det} \phi|
$$

which is a positive real number independent of the choice of $\phi$. We call it the Sinnott symbol of $A$ to $B$. Note that

(1) For lattices $A, B \subseteq V$, if $B \subseteq A$, then $(A: B)_{V}=\#(A / B)$.

(2) Given lattices $A, B, C \subseteq V$, then $(A: B)(B: C)=(A: C)$.

(3) Let $f: V_{1} \rightarrow V_{2}$ be an isomorphism of vector spaces. Let $A$ and $B$ be lattices in $V_{1}$. Then $(A: B)_{V_{1}}=(f(A): f(B))_{V_{2}}$.

For more results about the Sinnott symbol, see Sinnott [7] and [8].

2.2. The regulator map $\operatorname{reg}(A, B, \lambda)$. Given a finitely generated abelian group $A$, we denote the tensor product $A \otimes \mathbb{F}$ by $\mathbb{F} A$. Suppose we are given two finitely generated abelian groups $A$ and $B$, and an $\mathbb{F}$-linear isomorphism $\lambda: \mathbb{F} A \stackrel{\sim}{\rightarrow} B$. Choose free abelian subgroups $A^{\prime} \subseteq A$ and $B^{\prime} \subseteq B$ of finite index. Then $A^{\prime}$ and $B^{\prime}$ are of the same rank and hence isomorphic. Choose any isomorphism $\phi: B^{\prime} \stackrel{\sim}{\rightarrow} A^{\prime}$; it can be naturally extended to an isomorphism $\mathbb{F} \phi: \mathbb{F} B^{\prime} \stackrel{\sim}{\rightarrow} \mathbb{F} A^{\prime}$. Make the evident identification $\mathbb{F} A^{\prime}=\mathbb{F} A$ and $\mathbb{F} B^{\prime}=\mathbb{F} B$. Now put

$$
\operatorname{reg}(A, B, \lambda):=\frac{|\operatorname{det} \mathbb{F} \phi \circ \lambda| \cdot \# B / B^{\prime}}{\# A / A^{\prime}},
$$

which is a positive real number independent of the choice of $A^{\prime}, B^{\prime}$ and $\phi$. We call $\operatorname{reg}(A, B, \lambda)$ the regulator of $\lambda$ with respect to $A$ and $B$. We often write it $\operatorname{reg} \lambda$ in abbreviation. Here we calculate a few examples of the regulator:

(1) If both $A$ and $B$ are finite, then $\operatorname{reg}(A, B, 0)=\# B / \# A$.

(2) Let $f: A \rightarrow B$ be any homomorphism of finitely generated abelian groups with finite kernel and cokernel. Then $\operatorname{reg}(A, B, \mathbb{F} f)$ is \# coker $f / \# \operatorname{ker} f$.

(3) Let $A, B$ and $C$ be finitely generated abelian groups. Let $\lambda: \mathbb{F} A \sim \mathbb{F} B$ and $\mu: \mathbb{F} B \stackrel{\sim}{\rightarrow} \mathbb{F} C$ be $\mathbb{F}$-linear isomorphisms. Then $\operatorname{reg} \mu \circ \lambda=\operatorname{reg} \mu \cdot \operatorname{reg} \lambda$. 
(4) Let $V$ be a finite dimensional $\mathbb{F}$-vector space. Let $A, B \subseteq V$ be lattices. Let $\alpha: \mathbb{F} A \stackrel{\sim}{\rightarrow} V$ and $\beta: \mathbb{F} B \stackrel{\sim}{\rightarrow} V$ be the natural isomorphisms induced by the inclusions $A \subseteq V$ and $B \subseteq V$ respectively. Then $\operatorname{reg}\left(A, B, \beta^{-1} \circ \alpha\right)=$ $(B: A)_{V}$.

2.3. Passing to cohomology. Consider bounded complexes of finitely generated abelian groups $\left(A, d_{A}\right),\left(B, d_{B}\right)$ and an isomorphism $\lambda: \mathbb{F} A \stackrel{\sim}{\rightarrow} B$. $\lambda$ naturally induces an isomorphism $H^{i}(\lambda): H^{i}(\mathbb{F} A) \stackrel{\sim}{\rightarrow} H^{i}(\mathbb{F} B)$ for every degree $i$. Note also that $\mathbb{F} H^{i}(A)=H^{i}(\mathbb{F} A)$ and $\mathbb{F} H^{i}(B)=H^{i}(\mathbb{F} B)$. Then we have

Proposition 2.1. With the hypotheses above, then

$$
\prod_{i}\left(\operatorname{reg} \lambda^{i}\right)^{(-1)^{i}}=\prod_{i}\left(\operatorname{reg} H^{i}(\lambda)\right)^{(-1)^{i}} .
$$

Proof. First we claim that there exist subcomplexes $A^{\prime} \subseteq A$ and $B^{\prime} \subseteq B$ satisfying the following conditions:

(1) $A^{\prime i}$ and $B^{\prime i}$ are free abelian groups of the same rank as $A^{i}$ for all $i$.

(2) $H^{i}\left(A^{\prime}\right)$ and $H^{i}\left(B^{\prime}\right)$ are torsion free for all $i$.

(3) $A^{\prime}$ and $B^{\prime}$ are isomorphic complexes of abelian groups.

(4) The sequences

$$
\begin{aligned}
& 0 \rightarrow H^{i}\left(A^{\prime}\right) \rightarrow H^{i}(A) \rightarrow H^{i}\left(A / A^{\prime}\right) \rightarrow 0 \\
& 0 \rightarrow H^{i}\left(B^{\prime}\right) \rightarrow H^{i}(B) \rightarrow H^{i}\left(B / B^{\prime}\right) \rightarrow 0
\end{aligned}
$$

are exact for all $i$.

To prove the claim, let's first construct $A^{\prime}$ satisfying (1) and (2). Without loss of generality we suppose that $A^{i}=0$ for $i>0$. Consider the subgroup $d_{A}\left(A^{-1}\right)$ of $A^{0}$. Let $\left\{e_{1}, \cdots, e_{t}\right\}$ be a maximal independent set in $d_{A}\left(A^{-1}\right)$. Enlarge it to a maximal independent set $E_{0}$ of $A^{0}$ and let $A^{\prime 0}$ be the subgroup generated by $E_{0}$. Then $A^{0} / A^{\prime 0}$ is finite. Now for each $i, 1 \leq i \leq t$, find $f_{i} \in A^{-1}$ such that $d_{A}\left(f_{i}\right)=e_{i}$. Find a maximal independent set in $d_{A}\left(A^{-2}\right)$ and enlarge it to a maximal independent set $E_{1}$ in $\operatorname{ker}\left(d_{A}: A^{-1} \rightarrow A^{0}\right) . E_{1}$ and $\left\{f_{1}, \cdots, f_{t}\right\}$ are independent to each other in $A^{-1}$ and the subgroup generated by the union is of full rank in $A^{-1}$. Let it be $A^{\prime-1}$. Continuing this process, we obtain a subcomplex $A^{\prime}$ of $A$ which satisfies (1) and (2).

Similarly we construct a subcomplex $B^{\prime}$ of $B$ satisfying (1) and (2). But (3) and (4) follow easily from (1) and (2). Hence we proved the above claim. Now choose an isomorphism $\phi: B^{\prime} \rightarrow A^{\prime}$ of complexes. We have

$$
\begin{aligned}
\prod_{i}\left(\operatorname{reg} \lambda^{i}\right)^{(-1)^{i}} & =\prod_{i}\left(\frac{\left|\operatorname{det} \mathbb{F} \phi^{i} \circ \lambda^{i}\right| \cdot \#\left(B / B^{\prime}\right)^{i}}{\#\left(A / A^{\prime}\right)^{i}}\right)^{(-1)^{i}} \\
& =\prod_{i}\left(\frac{\left|\operatorname{det} \mathbb{F} H^{i}(\phi) \circ H^{i}(\lambda)\right| \cdot \# H^{i}\left(B / B^{\prime}\right)}{\# H^{i}\left(A / A^{\prime}\right)}\right)^{(-1)^{i}} \\
& =\prod_{i}\left(\operatorname{reg} H^{i}(\lambda)\right)^{(-1)^{i}} .
\end{aligned}
$$

Here we use the facts: (1) If $A$ is a complex of finite abelian groups, then

$$
\prod_{i}\left(\# H^{i}(A)\right)^{(-1)^{i}}=\prod_{i}\left(\# A^{i}\right)^{(-1)^{i}} .
$$


(2) If $V$ is a complex of $\mathbb{F}$-vector spaces and $\phi$ is an automorphism of $V$, then

$$
\prod_{i}\left|\operatorname{det} \phi^{i}\right|^{(-1)^{i}}=\prod_{i}\left|\operatorname{det} H^{i}(\phi)\right|^{(-1)^{i}} .
$$

\section{The UnIVERSAL NORM DISTRIBUtion}

3.1. Definition. Let $r$ be a positive integer. We call the factor $f \mid r$ a stalk of $r$ if $f$ and $r / f$ are prime to each other, i.e., $\left(f, \frac{r}{f}\right)=1$. For every factor $f$ of $r$, we denote by $\tilde{f}$ the smallest stalk of $r$ dividing $f$. In particular, $\tilde{p}$ is the largest $p$-power dividing $r$. We denote by $\left.f\right|_{s} r$ if $f$ is a stalk of $r$. For $\left.f\right|_{s} r$, we define $\operatorname{deg} f$ as the number of prime factors of $f$. Let

$$
T_{r}^{\prime}:=\left\{\frac{a}{r} \in \frac{1}{r} \mathbb{Z} / \mathbb{Z}:(a, r)=1\right\}, \quad T_{r}=\bigcup_{\left.f\right|_{s} r} T_{f}^{\prime} .
$$

We let $\mathbf{A}_{r}$ be the free abelian group with basis of symbols $\left\{[a]: a \in T_{r}\right\}$. For any $\sigma_{t} \in G_{r}=\operatorname{Gal}\left(\mathbb{Q}\left(\mu_{r}\right) / \mathbb{Q}\right)$, set $\sigma_{t}([a])=[t a]$; this makes $\mathbf{A}_{r}$ a $G_{r}$-module. Moreover, the submodule $\mathbf{A}_{f}^{\prime}$ generated by $\left\{[a]: a \in T_{f}^{\prime}\right\}$ is a free $\mathbb{Z}\left[G_{f}\right]$ of rank 1 . We regard $G_{f}$ as the subgroup $\operatorname{Gal}\left(\mathbb{Q}\left(\mu_{r}\right) / \mathbb{Q}\left(\mu_{r / f}\right)\right)$ of $G_{r}$ and denote by $N_{f} \in Z\left[G_{r}\right]$ the norm of $G_{f}$.

For any prime $p \mid r$, let $\operatorname{Fr}_{p}$ be the Frobenius of $p$ in $G_{r / \tilde{p}} \subseteq G_{r}$. Let $\mathbf{n}=$ $\{\mathbf{n}(p ; x)\}_{p \mid r}$ where $\mathbf{n}(p ; x)$ is a polynomial in $x$ with integer coefficients. Let

$$
X_{\tilde{p}}\left[\frac{a}{f}\right]:=\mathbf{n}\left(p ; \operatorname{Fr}_{p}^{-1}\right)\left[\frac{a}{f}\right]-N_{\tilde{p}}\left[\frac{a}{f \tilde{p}}\right],\left.\quad f\right|_{s} r, \quad p \nmid f,(a, f)=1,
$$

be a $G_{r}$-operator from $\mathbf{A}_{r / \tilde{p}}$ to $\mathbf{A}_{r}$. Moreover, let $X_{1}=1$ and for every $1 \neq\left. g\right|_{s} r$, let $X_{g}:=\prod_{p \mid g} X_{\tilde{p}}$, which is a $G_{r}$-operator from $\mathbf{A}_{r / g}$ to $\mathbf{A}_{r}$. Let $D_{\mathbf{n}, r}$ be the $G_{r}$-submodule generated by $X_{\tilde{p}} \mathbf{A}_{r / \tilde{p}}$ for all primes $p \mid r$ and let $U_{\mathbf{n}, r}=\mathbf{A}_{r} / D_{\mathbf{n}, r}$. We call $U_{\mathbf{n}, r}$ the universal norm distribution of level $r$ defined by $\mathbf{n}$. In brevity, we call $\mathbf{n}$ a norm distribution.

3.2. Basic properties. For any $x \in \mathbb{Q} / \mathbb{Z}$, let $r$ be its order. Then one can uniquely write

$$
x \equiv \sum_{p \mid r} \frac{x_{p}}{\tilde{p}} \quad \bmod \mathbb{Z}, \quad 0<x_{p}<\tilde{p}, p \nmid x_{p} .
$$

Say $x \in B_{n}$ if there exist exactly $n$ primes $p$ such that $x_{p}=1$ (assume $0 \in B_{0}$ ). Thus we make $\mathbb{Q} / \mathbb{Z}$ the disjoint union of $B_{n}$ for $n \geq 0$. We have

Proposition 3.1. (i) $\mathbf{A}_{r}$ possesses a $\mathbb{Z}$-basis

$$
\left\{X_{f}[a]: a \in T_{r / f} \cap B_{0},\left.f\right|_{s} r\right\} .
$$

(ii) For any $\mathbf{n}, U_{\mathbf{n}, r}$ is a free abelian group of rank $\left|G_{r}\right|$ with a basis $\{[a]: a \in$ $\left.T_{r} \cap B_{0}\right\}$.

(iii) If $r$ is $2 \bmod 4$, then $U_{\mathbf{n}, r}=U_{\mathbf{n}, r / 2}$.

(iv) If for all $p \mid r$, one has $\mathbf{n}(p ; x)=1-x$, then $U_{\mathbf{n}, r}=U_{r}$, the universal ordinary distribution of level $r$.

(v) If for all $p \mid r, \mathbf{n}(p ; x)=1$, then $U_{\mathbf{n}, r}=\mathbb{Z}\left[G_{r}\right]$. 
Proof. (i) On one hand, the number of elements in this set is

$$
\sum_{\left.f\right|_{s} r} \sum_{\left.g\right|_{s} \frac{r}{f}} \prod_{p \mid g}\left(\left|N_{\tilde{p}}\right|-1\right)=\prod_{p \mid r}\left(\left|N_{\tilde{p}}\right|+1\right)
$$

which is the $\mathbb{Z}$-rank of $\mathbf{A}_{r}$. On the other hand, if $x \in T_{r} \cap B_{n}$ for $n>0$, suppose that $x \in T_{g}^{\prime}$ and let $p \mid g$ be a prime for which $x_{p}=1$. Then

$$
[x]=-X_{\tilde{p}}[\tilde{p} x]+\mathbf{n}\left(p ; \mathrm{Fr}_{p}^{-1}\right)[\tilde{p} x]-\left(N_{\tilde{p}}-1\right)[x] .
$$

This identity tells us that

$$
\mathbf{A}_{r} \cap\left\langle B_{n}\right\rangle \subseteq \sum_{p \mid r} X_{\tilde{p}} \mathbf{A}_{r / \tilde{p}}+\sum_{p \mid r} \mathbf{A}_{r / \tilde{p}}+\mathbf{A}_{r} \cap\left\langle B_{n-1}\right\rangle
$$

where $\left\langle B_{n}\right\rangle$ denotes the free abelian group generated by $B_{n}$. Now by induction, the given set generates $\mathbf{A}_{r}$.

(ii) Clearly from (i).

(iii) This follows immediately from the fact that for any odd $f$,

$$
\left[\frac{1}{2 f}\right]=\mathbf{n}\left(2 ; \operatorname{Fr}_{2}^{-1}\right)\left[\frac{1}{f}\right]-X_{2}\left[\frac{1}{f}\right]
$$

(iv) For $\mathbf{n}(p ; x)=1-x$, recall that $U_{r}$ is the quotient of $\tilde{\mathbf{A}}_{r}=\left\langle[a]: a \in \frac{1}{r} \mathbb{Z} / \mathbb{Z}\right\rangle$ by relations

$$
[a]-\sum_{p b=a}[b], \quad a \in \frac{p}{r} \mathbb{Z} / \mathbb{Z} .
$$

One defines $\pi: \tilde{\mathbf{A}}_{r} \rightarrow U_{\mathbf{n}, r}$ to be the $G_{r}$-homomorphism by

$$
\left[\frac{1}{f}\right] \longmapsto \sum_{0 \leq a \leq \frac{\tilde{f}}{f}-1}\left[\frac{a f+1}{\tilde{f}}\right] .
$$

Certainly $\pi$ is surjective. One can check $\pi$ factors through $U_{r}$. But $U_{r}$ and $U_{\mathbf{n}, r}$ have the same rank as free abelian groups, thus $\pi$ induces an isomorphism.

(v) We define a $G_{r}$ homomorphism $\pi^{\prime}: \mathbf{A}_{r} \rightarrow \mathbb{Z}\left[G_{r}\right]$ by sending $\left[\frac{1}{f}\right]$ to $N_{r / f}$ for each $\left.f\right|_{s} r$. It is easy to check that $\pi^{\prime}$ is surjective and factors through $U_{\mathbf{n}, r}$ for $\mathbf{n}(p ; x)=1$. Now by (ii), $\pi^{\prime}$ is an isomorphism from $U_{\mathbf{n}, r}$ to $\mathbb{Z}\left[G_{r}\right]$.

Remark 3.2. (1) In the sequel, we assume that $r$ is not $2 \bmod 4$.

(2) We call $\mathbf{n}$ ordinary if $\mathbf{n}(p ; x)=1-x$ for all $p \mid r$. We call $\mathbf{n}$ trivial if $\mathbf{n}(p ; x)=1$ for all $p \mid r$. Another important norm distribution is $\mathbf{n}(2, x)=0$ and $\mathbf{n}(p ; x)=-x$ for $p \neq 2$. In this case $U_{\mathbf{n}, r}$ is isomorphic to the additive integer ring $\mathcal{O}_{\mathbb{Q}\left(\mu_{r}\right)}$. Following Anderson, we call it the universal predistribution.

3.3. Anderson's resolution of $U_{\mathbf{n}, r}$. For any $\left.g\right|_{s} r$ and $p \mid r$, we let $\omega(p, g)=$ $(-1)^{j-1}$ if $p$ is the $j$-th smallest prime factor of $g$ and let $\omega(p, g)=0$ if $p \nmid g$. Let $L_{r}$ be the free abelian group generated by symbols

$$
\left\{[a, g]: a \in T_{r / g},\left.g\right|_{s} r\right\} .
$$

We set $\operatorname{deg}[a, g]=-\operatorname{deg} g=-|\{p: p \mid g\}|$ and set $\sigma_{t}[a, g]=[t a, g]$; thus $L_{r}$ becomes a graded $G_{r}$-module. We write it as $L_{r}^{\bullet}$. Given a distribution $\mathbf{n}$; one lets

$$
d_{\mathbf{n}}\left[\frac{1}{f}, g\right]=\sum_{p \mid g} \omega(p, g)\left(\mathbf{n}\left(p ; \operatorname{Fr}_{p}^{-1}\right)\left[\frac{1}{f}, \frac{g}{\tilde{p}}\right]-N_{\tilde{p}}\left[\frac{1}{f \tilde{p}}, \frac{g}{\tilde{p}}\right]\right)
$$


and extends it by $G_{r}$-action. One can check that $d_{\mathbf{n}}^{2}=0$. Thus $d_{\mathbf{n}}$ is a differential of degree 1.

Theorem 3.3. One has $H^{n}\left(L_{r}^{\bullet}, d_{\mathbf{n}}\right)=0$ for $n \neq 0$. For $n=0, H^{0}\left(L_{r}^{\bullet}, d_{\mathbf{n}}\right)$ is isomorphic to $U_{\mathbf{n}, r}$ by $[x, 1] \mapsto[x]$.

Proof. This is a theorem of Anderson in the case of universal ordinary distribution. One may check Appendix A of Ouyang [6] for the proof. The general case has no extra difficulty.

Remark 3.4. By this theorem, we call the underlying module $L_{r}^{\bullet}$ Anderson's module of level $r$ and $\left(L_{r}^{\bullet}, d_{\mathbf{n}}\right)$ Anderson's resolution of $U_{\mathbf{n}, r}$.

Corollary 3.5. The $\mathbb{Q}\left[G_{r}\right]$-module $\mathbb{Q} \otimes_{\mathbb{Z}} U_{\mathbf{n}, r}$ is a free $\mathbb{Q}\left[G_{r}\right]$-module of rank 1 .

Proof. One studies the characters of $\mathbb{Q}\left[G_{r}\right]$-representations in $\mathbb{Q} \otimes L_{r}$.

3.4. The $J$-cohomology of $U_{\mathbf{n}, r}$. Let $J=\{1, c\} \subset G_{r}$ where $c$ is the complex conjugation. As an application of Theorem [3.3, we compute the Tate cohomology $\hat{H}\left(J, U_{\mathbf{n}, r}\right)$ by using Anderson's resolution $\left(L_{r}, d_{\mathbf{n}}\right)$. We study the spectral sequence

$$
E_{2}^{p, q}=H_{d_{\mathbf{n}}}^{p}\left(\hat{H}^{q}\left(J, L_{r}^{\bullet}\right)\right) \Rightarrow \hat{H}^{p+q}\left(J, U_{\mathbf{n}, r}\right) .
$$

For $f, g$ stalks of $r$ such that $(f, g)=1$, we write $\left[T_{f}^{\prime}, g\right]$ as the $G_{r}$-submodule generated by $\left\{[a, g]: a \in T_{f}^{\prime}\right\}$. Then $\left[T_{f}^{\prime}, g\right]$ is a free $\mathbb{Z}[J]$-module if $f \neq 1$, thus $\hat{H}^{q}\left(J,\left[T_{f}^{\prime}, g\right]\right)=0$ for $f \neq 1$. For $f=1$, if $q$ is odd, we still get $\hat{H}^{q}\left(J,\left[T_{f}^{\prime}, g\right]\right)=0$. For $q$ even, we get one copy of $\mathbb{Z} / 2 \mathbb{Z}$. Thus for $q$ even, the complex $\hat{H}^{q}\left(J, L_{r}^{\bullet}\right)$ is the graded complex

$$
\bigoplus_{\left.g\right|_{s} r} \mathbb{Z} / 2 \mathbb{Z}[g], \quad \operatorname{deg}[g]=-\operatorname{deg} g,
$$

and the differential $\bar{d}_{\mathbf{n}}$ induced by $d_{\mathbf{n}}$ is given by

$$
\bar{d}_{\mathbf{n}}[g]=\sum_{p \mid g} \omega(p, g) \mathbf{n}(p ; 1)[g / \tilde{p}] .
$$

If all $\mathbf{n}(p ; 1)$ are even, then $\bar{d}_{\mathbf{n}}=0$. Now suppose that there is a prime $p_{1} \mid r$ such that $\mathbf{n}\left(p_{1} ; 1\right)$ is odd. Let $\bar{d}_{\mathbf{n}, p}:[g] \mapsto \omega(p, g) \mathbf{n}(p ; 1)[g / \tilde{p}]$. Then $\bar{d}_{\mathbf{n}}=\sum_{p \mid r} \bar{d}_{\mathbf{n}, p}$. One checks that $\bar{d}_{\mathbf{n}, p}^{2}=0$ and $\bar{d}_{\mathbf{n}, p} \bar{d}_{\mathbf{n}, p^{\prime}}+\bar{d}_{\mathbf{n}, p^{\prime}} \bar{d}_{\mathbf{n}, p}=0$ for $p, p^{\prime}$ prime factors of $r$. The assumption that $\mathbf{n}\left(p_{1} ; 1\right)$ is odd means that the complex $\hat{H}^{q}\left(J, L_{r}^{\bullet}\right)$ is actually $\bar{d}_{\mathbf{n}, p_{1}}$-acyclic and hence $\bar{d}_{\mathbf{n}}$-acyclic. In conclusion, one has

Proposition 3.6. For the spectral sequence $H_{d_{\mathbf{n}}}^{p}\left(\hat{H}^{q}\left(J, L_{r}^{\bullet}\right)\right) \Rightarrow \hat{H}^{p+q}\left(J, U_{\mathbf{n}, r}\right)$, one has

(i) If for all $p \mid r, \mathbf{n}(p ; 1)$ is even, then

$$
E_{1}^{p, q}=E_{2}^{p, q}= \begin{cases}\bigoplus_{\operatorname{deg} g=-p} \mathbb{Z} / 2 \mathbb{Z}[g], & \text { if q even, } \\ 0, & \text { if } q \text { odd. }\end{cases}
$$

In this case the spectral sequence degenerates at $E_{1} . \hat{H}^{n}\left(J, U_{\mathbf{n}, r}\right)=(\mathbb{Z} / 2 \mathbb{Z})^{2^{\operatorname{deg} r-1}}$ for $n \in \mathbb{Z}$.

(ii) If there exists a prime $p \mid r$ with $\mathbf{n}(p ; 1)$ odd, then $E_{2}^{p, q}=0$ for all $p, q$. In this case $\hat{H}^{n}\left(J, U_{\mathbf{n}, r}\right)=0$. 
Proof. The only thing we need to prove is the degeneration of $E_{1}$-terms of the spectral sequence in case (i). In that case, if we let $S L_{r}^{\bullet}$ be the submodule of $L_{r}^{\bullet}$ generated by $[a, g], a \in T_{r / g} \backslash\{0\}$ for all $\left.g\right|_{s} r$. Then $S L^{\bullet}$ is $d_{\mathbf{n}^{-}}$stable and the quotient module $Q L_{r}^{\bullet}$ has induced differential 0 . Hence the spectral sequence $H_{0}^{p}\left(\hat{H}^{q}\left(J, Q L_{r}^{\bullet}\right)\right)$ degenerates at $E_{1}$. We have $\hat{H}^{q}\left(J, L_{r}^{\bullet}\right) \cong \hat{H}^{q}\left(J, Q L_{r}^{\bullet}\right)$, i.e., the $E_{1}^{p, q}$-terms of the two spectral sequences are isomorphic. Basic theory of spectral sequence tells us the degeneration of $H_{d_{\mathbf{n}}}^{p}\left(\hat{H}^{q}\left(J, L_{r}^{\bullet}\right)\right) \Rightarrow \hat{H}^{n}\left(J, U_{\mathbf{n}, r}\right)$ at $E_{1}$.

Remark 3.7. (1) If for all $p \mid r, \mathbf{n}(p ; 1)$ is even, we call $\mathbf{n}$ of $J$-type I; if there exists $p \mid r$ such that $\mathbf{n}(p ; 1)$ is odd, we call $\mathbf{n}$ of $J$-type II.

(2) For the universal ordinary distribution, the results in this section are wellknown; see for example, Kubert [4, 5] and Washington [9]. Our approach here follows the ideas from Anderson [1, 3].

\section{INDEX CALCULATION}

4.1. The connecting homomorphism $\phi_{\mathbf{n}_{1}, \mathbf{n}_{2}, r}$. Given two distributions $\mathbf{n}_{1}$ and $\mathbf{n}_{2}$, we define a $G_{r}$-automorphism of $\mathbb{Q} \otimes L_{r}^{\bullet}:=V_{r}^{\bullet}$ by

$$
\phi_{\mathbf{n}_{1}, \mathbf{n}_{2}, r}:\left[\frac{1}{f}, g\right] \longmapsto \sum_{\left.f^{\prime}\right|_{s} f}(-1)^{\operatorname{deg} f^{\prime}} \prod_{p \mid f^{\prime}} \frac{\mathbf{n}_{2}\left(p ; \mathrm{Fr}_{p}^{-1}\right)-\mathbf{n}_{1}\left(p ; \mathrm{Fr}_{p}^{-1}\right)}{\left|N_{\tilde{p}}\right|}\left[\frac{f^{\prime}}{f}, g\right] .
$$

Immediately one sees that the determinant of $\phi_{\mathbf{n}_{1}, \mathbf{n}_{2}, r}$ is 1 when restricting to every grade component of $V_{r}^{\bullet}$. By straightforward calculation, one sees that $\phi_{\mathbf{n}_{2}, \mathbf{n}_{1}, r}$ is the inverse of $\phi_{\mathbf{n}_{1}, \mathbf{n}_{2}, r}$ and

$$
\phi_{\mathbf{n}_{1}, \mathbf{n}_{2}, r} d_{\mathbf{n}_{1}}=d_{\mathbf{n}_{2}} \phi_{\mathbf{n}_{1}, \mathbf{n}_{2}, r} .
$$

This homomorphism thus induces an isomorphism from $\mathbb{Q} \otimes U_{\mathbf{n}_{1}, r}$ to $\mathbb{Q} \otimes U_{\mathbf{n}_{2}, r}$; we still write it as $\phi_{\mathbf{n}_{1}, \mathbf{n}_{2}, r}$. Then $\phi_{\mathbf{n}_{1}, \mathbf{n}_{2}, r}\left(U_{\mathbf{n}_{1}, r}\right)$ is a lattice in $\mathbb{Q} \otimes U_{\mathbf{n}_{2}, r}$. In particular, for $\mathbf{n}_{2}$ trivial, $\phi_{\mathbf{n}_{1}, \mathbf{n}_{2}, r}$ induces an isomorphism

$$
\begin{aligned}
\phi_{\mathbf{n}_{1}, r}: \mathbb{Q} \otimes U_{\mathbf{n}_{1}, r} & \longrightarrow \mathbb{Q}\left[G_{r}\right], \\
{\left[\frac{1}{f}\right] } & \longmapsto N_{r / f} \prod_{p \mid f}\left(1-\frac{\left(1-\mathbf{n}_{1}\left(p ; \mathrm{Fr}_{p}^{-1}\right)\right) N_{\tilde{p}}}{\left|N_{\tilde{p}}\right|}\right) .
\end{aligned}
$$

This way, we give an explicit proof of Corollary 3.5 Now if $\mathbf{n}_{1}$ is ordinary, then $\phi_{\mathbf{n}_{1}, r}\left(U_{r}\right)$ is nothing but the module $U$ introduced by Iwasawa (see Sinnott [7]).

4.2. A general index formula. For any $R=\mathbb{Z}\left[G_{r}\right]$-module $M$ and an arbitrary ideal $\theta$ of $R$, let $M^{\theta}$ be the submodule of $M$ annihilated by $\theta$.

Theorem 4.1. Let $\mathbf{n}_{1}$ and $\mathbf{n}_{2}$ be two norm distributions. Let $\theta$ be an arbitrary ideal of $\mathbb{Z}\left[G_{r}\right]$. Then

$$
\left(U_{\mathbf{n}_{2}, r}^{\theta}: \phi_{\mathbf{n}_{1}, \mathbf{n}_{2}, r}\left(U_{\mathbf{n}_{1}, r}^{\theta}\right)\right)=I\left(L_{r}^{\bullet}, d_{\mathbf{n}_{1}} ; \theta\right)^{-1} \cdot I\left(L_{r}^{\bullet}, d_{\mathbf{n}_{2}} ; \theta\right),
$$

where

$$
I\left(L_{r}^{\bullet}, d_{\mathbf{n}} ; \theta\right)=\frac{\# \operatorname{coker}\left(H^{0}\left(L_{r}^{\theta \bullet}, d_{\mathbf{n}}\right) \rightarrow H^{0}\left(L_{r}^{\bullet}, d_{\mathbf{n}}\right)^{\theta}\right)}{\# \operatorname{tor} H^{0}\left(L_{r}^{\theta \bullet}, d_{\mathbf{n}}\right) \cdot \prod_{i \neq 0} \# H^{i}\left(L_{r}^{\theta \bullet}, d_{\mathbf{n}}\right)^{(-1)^{i}}}
$$


Proof. In brevity we write $\phi=\phi_{\mathbf{n}_{1}, \mathbf{n}_{2}, r}$ in the proof. We apply Proposition 2.1] to the complexes $\left(L_{r}^{\theta \bullet}, d_{\mathbf{n}_{1}}\right),\left(L_{r}^{\theta \bullet}, d_{\mathbf{n}_{2}}\right)$ and the isomorphism $\left.\phi\right|_{V^{\theta} \boldsymbol{\bullet}}$. We'll extensively use the examples in $\S 2.2$. We have:

(1) $\operatorname{det}\left(\left.\phi\right|_{V^{\theta i}}\right)=1$ for all $i$. This follows immediately from the definition of $\phi$ : there exists a sequence of $\mathbb{Q}\left[G_{r}\right]$-modules $V_{0}^{i} \subseteq \cdots \subseteq V_{m}^{i}=V^{i}$ such that the map induced by $\phi$ in the quotient $V_{j}^{i} / V_{j-1}^{i}$ is the identity map.

(2) For all $i \neq 0$, since $H^{i}\left(V_{r}^{\theta \bullet}, d_{\mathbf{n}_{1}}\right)=H^{i}\left(V_{r}^{\theta \bullet}, d_{\mathbf{n}_{2}}\right)=0, H^{i}\left(L_{r}^{\theta \bullet}, d_{\mathbf{n}_{1}}\right)$ and $H^{i}\left(L^{\theta \bullet}, d_{\mathbf{n}_{2}}\right)$ are both finite and $H^{i}(\phi)=0$. We have

$$
\operatorname{reg}\left(H^{i}\left(L_{r}^{\theta \bullet}, d_{\mathbf{n}_{1}}\right), H^{i}\left(L_{r}^{\theta \bullet}, d_{\mathbf{n}_{2}}\right), H^{i}(\phi)\right)=\# H^{i}\left(L_{r}^{\theta}, d_{\mathbf{n}_{2}}\right) / \# H^{i}\left(L_{r}^{\theta \bullet}, d_{\mathbf{n}_{1}}\right) .
$$

(3) For $i=0$, for $j=1,2$, consider the maps $\alpha_{j}: H^{0}\left(L_{r}^{\theta}, d_{\mathbf{n}_{j}}\right) \rightarrow H^{0}\left(L_{r}^{\bullet}, d_{\mathbf{n}_{j}}\right)^{\theta}$. We have $H^{0}(\phi) \circ \mathbb{F} \alpha_{1}=\mathbb{F} \alpha_{2} \circ H^{0}(\phi)$. Then

$$
\begin{aligned}
& \operatorname{reg}\left(H^{0}\left(L_{r}^{\theta \bullet}, d_{\mathbf{n}_{1}}\right), H^{0}\left(L_{r}^{\theta \bullet}, d_{\mathbf{n}_{2}}\right), H^{0}(\phi)\right) \\
& \quad=\operatorname{reg}\left(\alpha_{1}\right) \cdot \operatorname{reg}\left(\alpha_{2}\right)^{-1} \cdot \operatorname{reg}\left(H^{0}\left(L_{r}^{\bullet}, d_{\mathbf{n}_{1}}\right)^{\theta}, H^{0}\left(L_{r}^{\bullet}, d_{\mathbf{n}_{2}}\right)^{\theta}, H^{0}(\phi)\right) .
\end{aligned}
$$

Now

$$
\operatorname{reg}\left(\alpha_{j}\right)=\frac{\# \operatorname{coker}\left(H^{0}\left(L_{r}^{\theta \bullet}, d_{\mathbf{n}_{j}}\right) \rightarrow H^{0}\left(L_{r}^{\bullet}, d_{\mathbf{n}_{j}}\right)^{\theta}\right)}{\# \operatorname{tor} H^{0}\left(L_{r}^{\theta \bullet}, d_{\mathbf{n}_{j}}\right)}
$$

and

$$
\operatorname{reg}\left(H^{0}\left(L_{r}^{\bullet}, d_{\mathbf{n}_{1}}\right)^{\theta}, H^{0}\left(L_{r}^{\bullet}, d_{\mathbf{n}_{2}}\right)^{\theta}, H^{0}(\phi)\right)=\left(U_{\mathbf{n}_{2}, r}^{\theta}: \phi U_{\mathbf{n}_{1}, r}^{\theta}\right) .
$$

Now by plugging in (1), (2) and (3) to Formula (2.2), we obtain the theorem.

In particular, if we let $\theta=0$, we obtain

Corollary 4.2. $\left(U_{\mathbf{n}_{2}, r}: \phi_{\mathbf{n}_{1}, \mathbf{n}_{2}, r} U_{\mathbf{n}_{1}, r}\right)=1$.

4.3. Study $I\left(L_{r}^{\bullet}, d_{\mathbf{n}} ; \theta\right)$ through spectral sequences. Let $M=R / \theta$ and let

$$
(P, \partial): \cdots \rightarrow P_{i} \rightarrow \cdots \rightarrow P_{1} \rightarrow P_{0} \rightarrow 0
$$

be a projective resolution of $M$. Let $K^{p, q}=\operatorname{Hom}_{G}\left(P_{q}, L_{r}^{p}\right)$, therefore we have a double complex $K^{\bullet \bullet \bullet}=\left(K^{p, q} ; d, \delta\right)$ with the differentials $d$ and $\delta$ induced by $d_{\mathbf{n}}$ and $\partial$ respectively. Let $K^{\bullet}$ be the total complex of $K^{\bullet \bullet \bullet}$. The two spectral sequences corresponding to the double complex $K^{\bullet \bullet \bullet}$ are

${ }^{\prime} E_{2}^{p, q}=H^{p}\left(\operatorname{Ext}_{G}^{q}\left(M, L_{r}^{\bullet}\right)\right) \Rightarrow H^{p+q}\left(K^{\bullet}\right),{ }^{\prime \prime} E_{2}^{p, q}=\operatorname{Ext}_{G}^{q}\left(M, H^{p}\left(L_{r}^{\bullet}\right)\right) \Rightarrow H^{p+q}\left(K^{\bullet}\right)$.

However, " $E_{2}^{p, q}=0$ for $p \neq 0$ and thus

$$
H^{i}\left(K^{\bullet}\right)=\operatorname{Ext}_{G}^{i}\left(M, U_{\mathbf{n}, r}\right) .
$$

Hence

$$
{ }^{\prime} E_{2}^{p, q}=H^{p}\left(\operatorname{Ext}_{G}^{q}\left(M, L_{r}^{\bullet}\right)\right) \Rightarrow \operatorname{Ext}_{G}^{p+q}\left(M, U_{\mathbf{n}, r}\right) .
$$

We drop the symbol ' from our notation from now on. Let $q=0$. Then

$$
E_{2}^{p, 0}=H^{p}\left(\operatorname{Ext}_{G}^{0}\left(M, L_{r}^{\bullet}\right)\right)=H^{p}\left(L_{r}^{\theta \bullet}\right) .
$$

Lemma 4.3. $E_{\infty}^{0,0}=\operatorname{im}\left(H^{0}\left(L_{r}^{\theta \bullet}\right) \rightarrow U_{\mathbf{n}, r}^{\theta}\right)$. 
Proof. We know the spectral sequence $E_{2}^{p, q}$ is from the filtration

$$
\mathrm{Fil}^{p} K^{\bullet \bullet \bullet}=\bigoplus_{p^{\prime} \geq p} K^{p^{\prime}, q}
$$

Because $\mathrm{Fil}^{1} K^{\bullet}$ is trivial, we have

$$
E_{\infty}^{0,0}=\operatorname{Fil}^{0} H^{0}\left(K^{\bullet}\right)=\operatorname{im}\left(H^{0}\left(\operatorname{Fil}^{0} K^{\bullet}\right) \rightarrow H^{0}\left(K^{\bullet}\right)\right) .
$$

Easy to see that $H^{0}\left(\mathrm{Fil}^{0} K^{\bullet}\right)=L_{r}^{0 \theta}$ and therefore

$$
E_{\infty}^{0,0}=\operatorname{im}\left(L_{r}^{0 \theta} \rightarrow U_{\mathbf{n}, r}^{\theta}\right) .
$$

Consider the following diagram with exact rows:

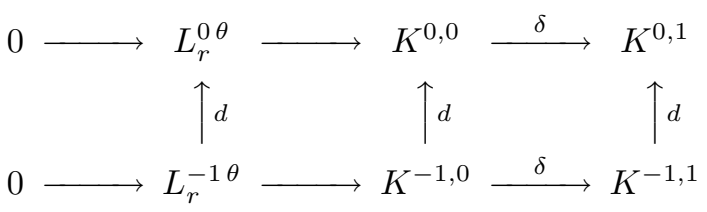

We see that $L_{r}^{-1 \theta}$ is contained in the boundary of $K^{0}=\bigoplus K^{p,-p}$. Furthermore, note that $H^{0}\left(L_{r}^{\theta \bullet}\right)=\operatorname{coker}\left(L_{r}^{-1 \theta} \rightarrow L_{r}^{0 \theta}\right)$; the lemma follows immediately.

Proposition 4.4. If one has

$$
\# \operatorname{Ext}_{G}^{1}\left(M, U_{\mathbf{n}, r}\right)=\prod_{q} \# H^{1-q}\left(\operatorname{Ext}_{G}^{q}\left(M, L_{r}^{\bullet}\right)\right),
$$

then

$$
I\left(L_{r}^{\bullet}, d_{\mathbf{n}} ; \theta\right)=\prod_{\substack{p+q \leq 0 \\ q>0}} \# H^{p}\left(\operatorname{Ext}_{G}^{q}\left(M, L_{r}^{\bullet}\right)\right)^{(-1)^{p+q}}=\prod_{\substack{p+q \leq 0 \\ q>0}}\left(\# E_{2}^{p, q}\right)^{(-1)^{p+q}} .
$$

Proof. First note that the given identity (4.3) is nothing but

$$
\prod_{q} \# E_{\infty}^{1-q, q}=\prod_{q} \# E_{2}^{1-q, q} .
$$

From the theory of the spectral sequence, $H^{\bullet}\left(E_{r}\right)=E_{r+1}$, then

$$
\# E_{2}^{p, q} \geq \# E_{3}^{p, q} \geq \cdots \geq \# E_{\infty}^{p, q} .
$$

Hence by (4.3),

$$
\# E_{2}^{1-q, q}=\# E_{3}^{1-q, q}=\cdots=\# E_{\infty}^{1-q, q},
$$

which means that for $r \geq 2$,

$$
\operatorname{im}\left(d_{r}: E_{r}^{1-q-r, q+r-1} \rightarrow E_{r}^{1-q, q}\right)=\operatorname{im}\left(d_{r}: E_{r}^{1-q, q} \rightarrow E_{r}^{1-q+r, q-r+1}\right)=0 .
$$

Therefore we have a shorter complex:

$$
\cdots \rightarrow E_{r}^{1-q-2 r, q+2 r-2} \rightarrow E_{r}^{1-q-r, q+r-1} \rightarrow 0 .
$$

Now we set to prove the following fact:

$$
\prod_{\substack{p+q \leq 0 \\(p, q) \neq(0,0)}}\left(\# E_{r}^{p, q}\right)^{(-1)^{p+q}} \cdot \# \text { tor } E_{r}^{0,0} \text { is independent of } r .
$$

Observe that in the set $\left\{E_{r}^{p, q}: p+q \leq 0, q \geq 0\right\}$, the only term not finite is $E_{r}^{0,0}$. If we substitute it by its torsion, we still get a group of complexes composed of finite abelian groups and with differential $d_{r}$. The cohomology groups are $E_{r+1}^{p, q}$ 
(or tor $E_{r+1}^{0,0}$ ). By the invariance of Euler characteristic under cohomology, (4.5) is proved. Note that $E_{\infty}^{0,0}$ is free and

$$
\prod_{\substack{p+q \leq 0 \\(p, q) \neq(0,0)}}\left(\# E_{\infty}^{p, q}\right)^{(-1)^{p+q}}=\# \operatorname{coker}\left(H^{0}\left(L_{r}^{\theta \bullet}\right) \rightarrow U_{\mathbf{n}, r}^{\theta}\right) .
$$

Formula 4.4 now follows immediately.

4.4. The index $\left(U_{\mathbf{n}_{2}, r}^{-}: \phi_{\mathbf{n}_{1}, \mathbf{n}_{2}, r} U_{\mathbf{n}_{1}, r}^{-}\right)$. In this case we have $\theta=1+c$. Let $M=\operatorname{coker}(\mathbb{Z}[J] \stackrel{1+c}{\rightarrow} \mathbb{Z}[J])$. Then $M$ has a projective resolution

$$
(P, \partial): \cdots \stackrel{\partial_{q+1}}{\longrightarrow} \mathbb{Z}[J] \stackrel{\partial_{q}}{\longrightarrow} \mathbb{Z}[J] \stackrel{\partial_{q-1}}{\longrightarrow} \cdots \stackrel{\partial_{0}}{\longrightarrow} \mathbb{Z}[J] \longrightarrow 0
$$

where $\partial_{q}=1+(-1)^{q} \cdot c$. Consider the spectral sequence

$$
H_{d_{\mathbf{n}}}^{p}\left(\operatorname{Ext}_{J}^{q}\left(M, L_{r}^{\bullet}\right)\right) \Rightarrow \operatorname{Ext}_{J}^{p+q}\left(M, U_{\mathbf{n}, r}\right) .
$$

One has $\operatorname{Ext}_{J}^{q}\left(M, L_{r}^{\bullet}\right)=\hat{H}^{q+1}\left(J, L_{r}^{\bullet}\right)$ for any $q>0$.

Proposition 4.5. The index $\left(U_{\mathbf{n}_{2}, r}^{-}: \phi_{\mathbf{n}_{1}, \mathbf{n}_{2}, r} U_{\mathbf{n}_{1}, r}^{-}\right)$is equal to

$$
\begin{cases}1, & \text { if } \mathbf{n}_{1} \text { and } \mathbf{n}_{2} \text { have the same J-type, } \\ 2^{-a}, & \text { if } \mathbf{n}_{1} \text { has J-type I, } \mathbf{n}_{2} \text { has J-type II, } \\ 2^{a}, & \text { if } \mathbf{n}_{1} \text { has J-type II, } \mathbf{n}_{2} \text { has J-type I, }\end{cases}
$$

where $a=1$ if $\operatorname{deg} r=1$ and $a=2^{\operatorname{deg} r-2}$ if $\operatorname{deg} r \geq 2$

Proof. The degeneration condition (4.3) in Proposition 4.4 is satisfied by Proposition [3.6 for both $d_{\mathbf{n}_{1}}$ and $d_{\mathbf{n}_{2}}$. By Proposition [3.6] and (4.4), it follows easily that $I\left(L_{r}^{\bullet}, d_{\mathbf{n}} ; 1+c\right)=1$ if $\mathbf{n}$ is of $J$-type II. If $\mathbf{n}$ is of $J$-type I, the power of 2 in $I\left(L_{r}^{\bullet}, d_{\mathbf{n}} ; 1+c\right)$ is then equal to

$$
\sum_{\substack{p+q \leq 0 \\
q>0 \text { odd }}}(-1)^{p+1}\left(\begin{array}{c}
\operatorname{deg} r \\
-p
\end{array}\right)= \begin{cases}1, & \text { if } \operatorname{deg} r=1 \\
2^{\operatorname{deg} r-2}, & \text { if } \operatorname{deg} r>1 .\end{cases}
$$

The Proposition now follows from Theorem 4.1

4.5. Recovery of Sinnott's calculation. We can now recover Sinnott's index calculation in [7]. By the corollary of Theorem 4.1] one has $(R: U)=1$. By Proposition 4.5. $\left(R^{-}: U^{-}\right)$is $\frac{1}{2}$ for $\operatorname{deg} r=1$ and $2^{-2^{\operatorname{deg} r-2}}$ for $\operatorname{deg} r \geq 2$. We also know that $U^{-} /(1-j) U=H^{1}(J, U)$, thus $\left(U^{-}:(1-j) U\right)=2^{2^{\operatorname{deg} r-1}}$. These indices are essentially what Sinnott needs in [7].

\section{REFERENCES}

[1] Anderson, Greg W., Another look at the index formulas of cyclotomic number theory, J. Number Theory 60(1996), 142-164. MR 97i:11108

[2] Anderson, Greg W., Index calculations by the double complex method, Working notes, 1998.

[3] Anderson, Greg W., A double complex for computing the sign-cohomology of the universal ordinary distribution. Recent Progress in Algebra (Taejonto/Seoul, 1997) 1-27, Contem. Math. 224, American Mathematical Society, Providence, 1999. MR 99k:11169

[4] Kubert, D.S., The universal ordinary distribution, Bull. Soc. Math. France 107(1979), 179202. MR 81b:12004

[5] Kubert, D.S., The $\mathbb{Z} / 2 \mathbb{Z}$ cohomology of the universal ordinary distribution, Bull. Soc. Math. France 107(1979), 203-224. MR 81a:20062 
[6] Ouyang, Y., Group cohomology of the universal ordinary distribution. J. reine. angew. Math. 537 (2001), 1-32.

[7] Sinnott, Warren, On the Stickelberger ideal and the circular units of a cyclotomic field. Annals of Mathematics 108(1978), 107-134. MR 58:5585

[8] Sinnott, Warren, On the Stickelberger ideal and the circular units of an abelian field. Invent. Math. 62(1980), 181-234. MR 82i:12004

[9] Washington, L.C., Introduction to cyclotomic fields, 2nd ed. Graduate Texts in Mathematics 83, Springer Verlag, New York, 1997. MR 97h:11130

Department of Mathematics, Univerity of Toronto, Toronto, Ontario, Canada M5S 3G3

E-mail address: youyang@math.toronto.edu 